ARTICLE

DOI: $10.1038 / s 41467-018-06503-8$

\title{
Tailoring manganese oxide with atomic precision to increase surface site availability for oxygen reduction catalysis
}

\author{
C. John Eom¹, Ding-Yuan Kuo ${ }^{1}$, Carolina Adamo ${ }^{2}$, Eun Ju Moon ${ }^{3}$, Steve J. May ${ }^{3}$, Ethan J. Crumlin (1) ${ }^{4}$, \\ Darrell G. Schlom ${ }^{1,5} \&$ Jin Suntivich ${ }^{1,5}$
}

Controlling the structure of catalysts at the atomic level provides an opportunity to establish detailed understanding of the catalytic form-to-function and realize new, non-equilibrium catalytic structures. Here, advanced thin-film deposition is used to control the atomic structure of $\mathrm{La}_{2 / 3} \mathrm{Sr}_{1 / 3} \mathrm{MnO}_{3}$, a well-known catalyst for the oxygen reduction reaction. The surface and sub-surface is customized, whereas the overall composition and $d$-electron configuration of the oxide is kept constant. Although the addition of $\mathrm{SrMnO}_{3}$ benefits the oxygen reduction reaction via electronic structure and conductivity improvements, $\mathrm{SrMnO}_{3}$ can react with ambient air to reduce the surface site availability. Placing $\mathrm{SrMnO}_{3}$ in the subsurface underneath a $\mathrm{LaMnO}_{3}$ overlayer allows the catalyst to maintain the surface site availability while benefiting from improved electronic effects. The results show the promise of advanced thin-film deposition for realizing atomically precise catalysts, in which the surface and sub-surface structure and stoichiometry are tailored for functionality, over controlling only bulk compositions.

\footnotetext{
${ }^{1}$ Department of Materials Science and Engineering, Cornell University, Ithaca, NY 14853, USA. ${ }^{2}$ Department of Applied Physics, Stanford University, Stanford, CA 94305, USA. ${ }^{3}$ Department of Materials Science and Engineering, Drexel University, Philadelphia, PA 19104, USA. ${ }^{4}$ Advanced Light Source, Lawrence Berkeley National Laboratory, Berkeley, CA 94720, USA. ${ }^{5}$ Kavli Institute at Cornell for Nanoscale Science, Cornell University, Ithaca, NY 14853, USA. Correspondence and requests for materials should be addressed to J.S. (email: jsuntivich@cornell.edu)
} 
U nderstanding the structure-activity relationship of a catalyst at the atomic level has been a long-standing challenge in the pursuit of a designer catalyst $\mathrm{t}^{1-5}$. Traditionally, researchers identify this relationship by varying the bulk structure and/or composition and examining the impact on the catalytic performance. Although these efforts have resulted in design principles and discoveries of new catalysts, options beyond bulk variable control have not been fully explored. Advances in non-platinum catalysts such as carbon-based and metal-oxide catalysts for alkaline conditions further accentuate the need for full exploration of catalyst design beyond bulk variable control ${ }^{6,7}$. An emerging concept is to independently tune the structure and composition in the surface and sub-surface layers, the layers most sensitive to surface reactions. Control of these parameters provide the allure of optimizing electronic structure and surface chemistry in separate but closely coupled atomic layers ${ }^{8,9}$. Independently controlling the surface and sub-surface structure and composition, however, requires the ability to prepare materials with atomic precisions in a non-equilibrium manner. This often presents a practical obstacle for materials scientists. The purpose of this article is to present a route to realize this concept via advanced thin-film deposition.

Studies of well-defined single crystals have shown that the subsurface atoms play an important role on the catalytic performance just as the surface atoms ${ }^{10-15}$. Driven by these findings, many researchers have synthesized catalysts with core-shell structures to manipulate the performance of the catalyst by using heat treatment to induce surface segregation or via post-synthetic deposition $^{16-19}$. These syntheses, however, do not afford the ability to control the surface and sub-surface structure and composition in a systematic fashion. As a result, it has not been straightforward to obtain the structure-activity relations from these experiments. The difficulty in controlling the surface and sub-surface structure and composition is particularly challenging for oxide catalysts used in alkaline fuel cells and metal-air batteries, where complex quaternary phase diagrams and hightemperature treatment used for preparations often push the catalyst to favor the equilibrium structure $20-26$.

Here, this difficulty is tackled through advanced thin-film deposition. Thin films of $\mathrm{La}_{2 / 3} \mathrm{Sr}_{1 / 3} \mathrm{MnO}_{3}$ are grown layer-bylayer by molecular beam epitaxy (MBE). The surface and subsurface compositions of the films were varied by tuning the deposition sequence while maintaining the overall stoichiometry and identical electronic structure. Comparison of the catalytic activity of the films for oxygen reduction reaction (ORR) show that the placement of the $\mathrm{SrMnO}_{3}$ layer in the sub-surface shows greater activity than when $\mathrm{SrMnO}_{3}$ is placed on the surface. Ambient pressure X-ray photoelectron spectroscopy (APXPS) reveals that the $\mathrm{SrMnO}_{3}$ layer improves the electronic structure and conductivity for ORR, but simultaneously reacts with ambient air to reduce the number of active sites. Placing $\mathrm{SrMnO}_{3}$ in the sub-surface is the optimal choice for the film to maximally benefit from the former effect, whereas minimizing the latter.

\section{Results}

Film growth and structural characterization. In all, 20-nm thick $\mathrm{La}_{2 / 3} \mathrm{Sr}_{1 / 3} \mathrm{MnO}_{3}$ heterostructures were grown by $\mathrm{MBE}$ on $\mathrm{TiO}_{2}-$ terminated (001) $\mathrm{SrTiO}_{3}$ single-crystal substrates ${ }^{27}$. (001) was chosen as the low-energy termination of the perovskite structure which allows for a layer-by-layer growth. The structural integrity of the heterostructures were characterized using fourcircle X-ray diffraction (XRD), angle-resolved photoemission spectroscopy (ARPES), and transport measurements ${ }^{28,29}$. These results also show that thin films studied in this article, thin films of low repeat units ( $n=1$ and 2), the $\mathrm{LaMnO}_{3}$ and $\mathrm{SrMnO}_{3}$ layers are commensurate to the substrate and the effects of interlayer strain are very small. For comparison, we study $\mathrm{LaMnO}_{3}$ (LLL) and

$\mathrm{La}_{2 / 3} \mathrm{Sr}_{1 / 3} \mathrm{MnO}_{3}$ (where $\mathrm{La}$ and $\mathrm{Sr}$ form a solid solution) films of the same thickness. For $\left(\mathrm{LaMnO}_{3}\right)_{2} /\left(\mathrm{SrMnO}_{3}\right)$, we permute the ordering between $\mathrm{LaMnO}_{3}$ and $\mathrm{SrMnO}_{3}$ to control the $\mathrm{SrMnO}_{3}$ placement at either the surface, sub-surface, and sub-sub-surface. Films are referred to be based on their repeat units: $\mathrm{SrMnO}_{3}-$ $\mathrm{LaMnO}_{3}-\mathrm{LaMnO}_{3}$ as "SLL", $\mathrm{LaMnO}_{3}-\mathrm{SrMnO}_{3}-\mathrm{LaMnO}_{3}$ as "LSL", and $\mathrm{LaMnO}_{3}-\mathrm{LaMnO}_{3}-\mathrm{SrMnO}_{3}$ as "LLS" (Fig. 1). In this nomenclature, the first letter of the name represents the layer that is closest to the surface. We follow the same naming convention for $\left(\mathrm{LaMnO}_{3}\right)_{4} /\left(\mathrm{SrMnO}_{3}\right)_{2}$, where we prepare LLSSLL and SSLLLL as our model $\left(\mathrm{LaMnO}_{3}\right)_{4} /\left(\mathrm{SrMnO}_{3}\right)_{2}$ heterostructures (Supplementary Figure 1). X-ray analysis shows reflections from both the perovskite structure and the superlattice (Fig. 2a and Supplementary Figure 2).

To verify the $\mathrm{SrMnO}_{3}$ placement, we measure the intensity ratios between the raw La $4 d$ and $\mathrm{Sr} 3 d$ peaks using APXPS. For excitation energies that correspond to probing depths of around one functional unit $(\sim 1.1 \mathrm{~nm})$ from the surface (photon energies of $340 \mathrm{eV}$ for $\mathrm{La} 4 d$ and $385 \mathrm{eV}$ for $\mathrm{Sr} 3 d$ ), the APXPS results show that LLS has the highest $\mathrm{La} / \mathrm{Sr}$ intensity ratio near the surface, followed by LSL, and then SLL (Fig. 2b). These compositional ratios agree with our intended $\mathrm{SrMnO}_{3}$ placement and are unchanged under different $\mathrm{pO}_{2}$ (Fig. 2b). The consistent compositional ratios support our naming nomenclature and our
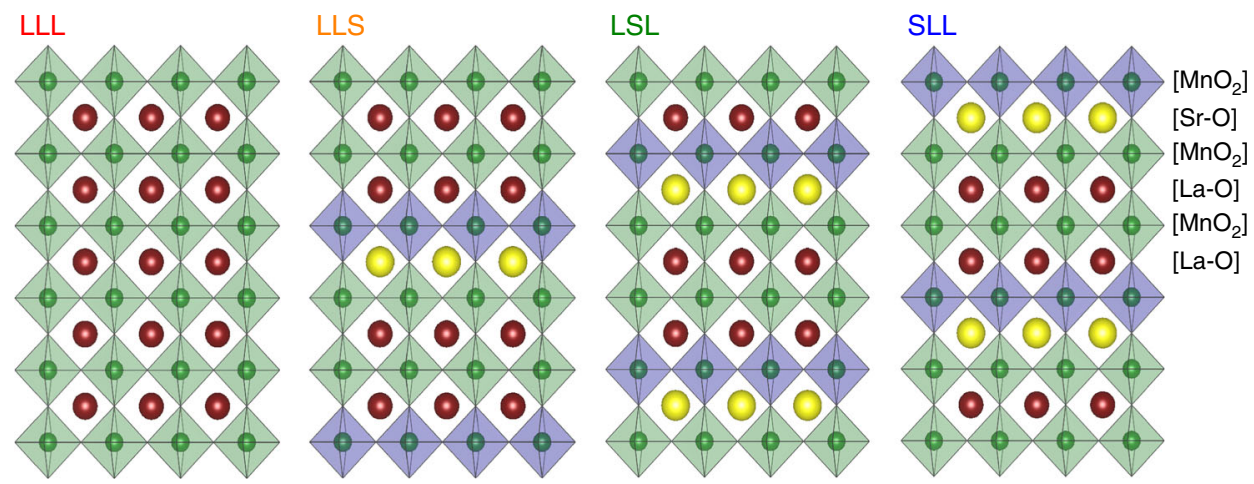

Fig. 1 Schematic illustrations of the $\left(\mathrm{LaMnO}_{3}\right)_{2} /\left(\mathrm{SrMnO}_{3}\right)$ heterostructures in this work. The naming scheme refers to the order of the A-site metals beginning at the surface layer. $\mathrm{LaMnO}_{3}-\mathrm{LaMnO}_{3}-\mathrm{LaMnO}_{3}$ as " $\mathrm{LLL}^{\prime}, \mathrm{LaMnO}_{3}-\mathrm{LaMnO}_{3}-\mathrm{SrMnO}_{3}$ as " $\mathrm{LLS}^{\prime}, \mathrm{LaMnO}_{3}-\mathrm{SrMnO}_{3}-\mathrm{LaMnO}_{3}$ as " $\mathrm{LSL}^{\prime}$, and SrMnO${ }_{3}-$ $\mathrm{LaMnO}_{3}-\mathrm{LaMnO}_{3}$ as "SLL." Blue manganite octahedra are associated with the $\mathrm{Sr}$-O layer (yellow) and the green manganite octahedra are associated with the La-O layer (maroon) 

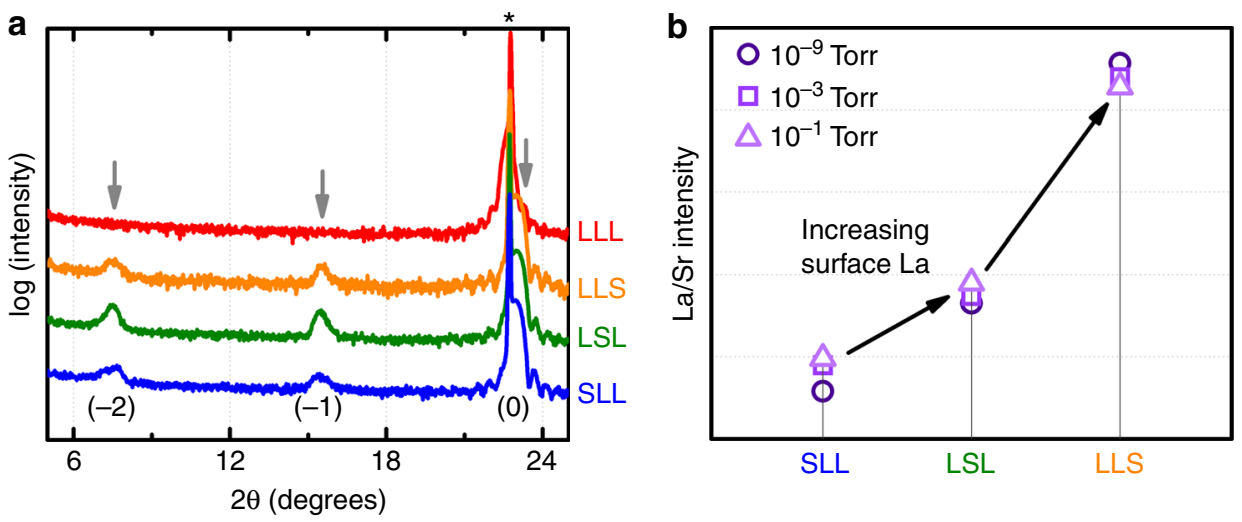

Fig. 2 Structure and near-surface composition of $\left(\mathrm{LaMnO}_{3}\right)_{2} /\left(\mathrm{SrMnO}_{3}\right)$ heterostructures. a X-ray diffraction patterns of $(\mathrm{LaMnO})_{2} /\left(\mathrm{SrMnO}_{3}\right)$ films. Peaks near $23^{\circ}$ can be assigned to the (001) pseudo-cubic perovskite structure and the substrate peak (003 and *, respectively). The peaks near $14^{\circ}$ and $7^{\circ}$ are assigned to the satellite reflections of the superlattice $(-1$ and -2$)$ from the cation ordering. $\mathbf{b}$ Ambient pressure X-ray photoelectron spectroscopy measurement of the $\mathrm{La} / \mathrm{Sr}$ intensity ratio of the $\left(\mathrm{LaMnO}_{3}\right)_{2} /\left(\mathrm{SrMnO}_{3}\right)$ heterostructures at different oxygen pressures. $\mathrm{LaMnO}_{3}-\mathrm{LaMnO}_{3}-\mathrm{LaMnO} \mathrm{O}_{3}$ is referred to as " $\mathrm{LLL}$ ", $\mathrm{LaMnO}_{3}-\mathrm{LaMnO}_{3}-\mathrm{SrMnO}_{3}$ as "LLS", $\mathrm{LaMnO}_{3}-\mathrm{SrMnO}_{3}-\mathrm{LaMnO}_{3}$ as "LSL", and $\mathrm{SrMnO}_{3}-\mathrm{LaMnO}_{3}-\mathrm{LaMnO}_{3}$ as "SLL"
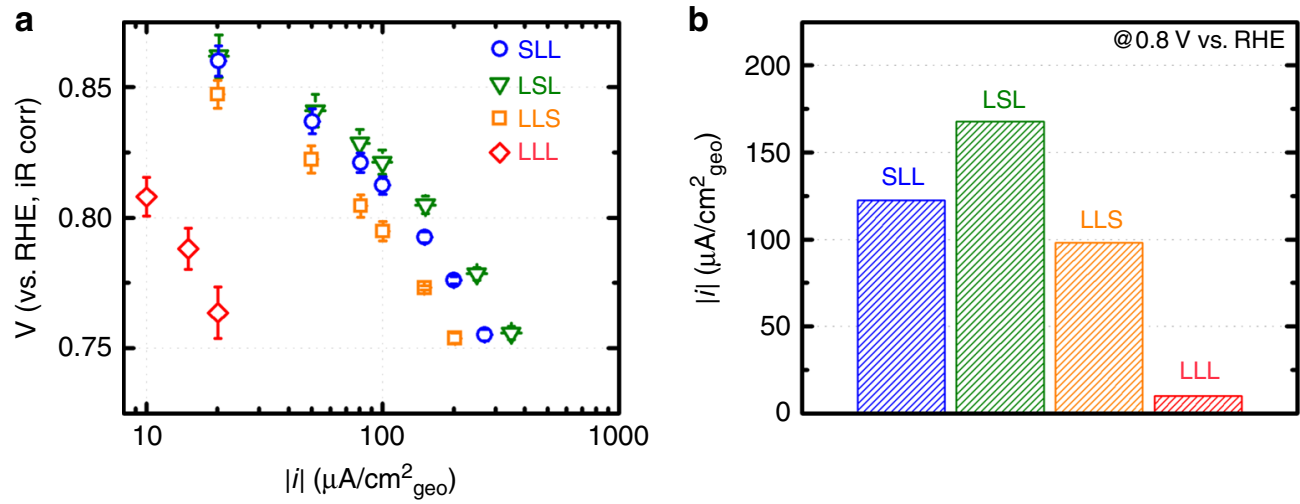

Fig. 3 Oxygen reduction reaction activities of $\left(\mathrm{LaMnO}_{3}\right)_{2} /\left(\mathrm{SrMnO}_{3}\right)$. a Tafel plot of the oxygen reduction reaction $(\mathrm{ORR})$ of $(\mathrm{LaMnO})_{2} /\left(\mathrm{SrMnO}_{3}\right)$ in $0.1 \mathrm{M}$ $\mathrm{KOH}$. Error bars represent standard deviations of three independent measurements using three pieces of films deposited under the same condition. $\mathbf{b} \mathrm{A}$ comparison of the ORR activities of $\left(\mathrm{LaMnO}_{3}\right)_{2} /\left(\mathrm{SrMnO}_{3}\right)$ at $0.8 \mathrm{~V}$ vs. reversible hydrogen electrode (RHE). $\mathrm{LaMnO}_{3}-\mathrm{LaMnO}_{3}-\mathrm{LaMnO} \mathrm{Mn}_{3}$ is referred to as "LLL", $\mathrm{LaMnO}_{3}-\mathrm{LaMnO}_{3}-\mathrm{SrMnO}_{3}$ as "LLS", $\mathrm{LaMnO}_{3}-\mathrm{SrMnO}_{3}-\mathrm{LaMnO}_{3}$ as "LSL", and $\mathrm{SrMnO}_{3}-\mathrm{LaMnO}_{3}-\mathrm{LaMnO}_{3}$ as "SLL"

assignment of any observed difference in the ORR activity to the $\mathrm{SrMnO}_{3}$ placement within the heterostructures.

ORR activity comparison. The surface and sub-surface placement plays an important role on the ORR. Figure 3 a shows the ORR activity on $\left(\mathrm{LaMnO}_{3}\right)_{2} /\left(\mathrm{SrMnO}_{3}\right)$ heterostructures in a Tafel plot. All $\left(\mathrm{LaMnO}_{3}\right)_{2} /\left(\mathrm{SrMnO}_{3}\right)$ heterostructures share the same Tafel slope, suggesting a similar reaction pathway (for the studied composition, the overall reaction is $\mathrm{O}_{2}+2 \mathrm{H}_{2} \mathrm{O}+4 \mathrm{e}^{-} \rightarrow 4 \mathrm{OH}^{-24}$ ). As our analysis focuses on the current range that is much smaller than the mass transport limit ${ }^{30}$, we did not apply any diffusion correction. For a visual comparison, Fig. $3 \mathrm{~b}$ shows the ORR current at $0.8 \mathrm{~V}$ vs. reversible hydrogen electrode (RHE). LSL, where the Sr layer resides in the sub-surface layer, is the most active, followed by SLL, LLS, and then LLL. The low activity of LLS suggests that Sr most efficiently benefits ORR when it is placed within two-unit cells from the surface $\mathrm{Mn}(<1 \mathrm{~nm})$. As $\mathrm{Sr}$ benefits ORR by affecting the $d$-electron configuration of $\mathrm{Mn}$ in the $e_{g}$ symmetry ${ }^{20}$, the low activity of LLS indicates that electronic effect of $\mathrm{Sr}$ is localized to approximately two-unit cells. This hypothesis is consistent with the observation that the ORR kinetics on $\left(\mathrm{LaMnO}_{3}\right)_{2} /\left(\mathrm{SrMnO}_{3}\right)$ is more active than
$\left(\mathrm{LaMnO}_{3}\right)_{4} /\left(\mathrm{SrMnO}_{3}\right)_{2}$; both LLSSLL and SSLLLL (Supplementary Figures 3,4) reveal that larger superlattice spacing impedes Sr from benefiting the topmost Mn layer for ORR. This interpretation of our results agrees with previous ARPES experiments and first-principle calculations. ARPES showed reduced electronic hopping integrals when the spacing between the $\mathrm{LaMnO}_{3}$ and $\mathrm{SrMnO}_{3}$ layers increases ${ }^{28}$. First-principle calculations showed that the charge transfer effects of the $\left(\mathrm{LaMnO}_{3}\right)_{2} /\left(\mathrm{SrMnO}_{3}\right)$ interfaces are confined to $\sim 2$-unit cells ${ }^{31,32}$. Comparing the electrochemical behavior of $\left(\mathrm{LaMnO}_{3}\right)_{2 n} /\left(\mathrm{SrMnO}_{3}\right) \mathrm{n}(n=1,2)$ to that of a random alloy ('Alloy') further reveals the effects of subsurface engineering (Supplementary Figure 5). The Alloy film, which should structurally represent a random mixture of the three $\left(\mathrm{LaMnO}_{3}\right)_{2} /\left(\mathrm{SrMnO}_{3}\right)$ films, performs close to the average of the three films, markedly poorer than LSL (Supplementary Figure 5). Both our results and those in the literature from the electronic structure community confirm a long-held intuition: sub-surface chemistry manipulation is the most direct way to influence the activity of the topmost catalytic layer, and that the effect gradually decreases as the manipulation occurs on a layer further away from the surface.

Having concluded the positive role of the near-surface $\mathrm{La}-\mathrm{Sr}$ interface, we can now discuss why ORR is more active when $\mathrm{Sr}$ is 

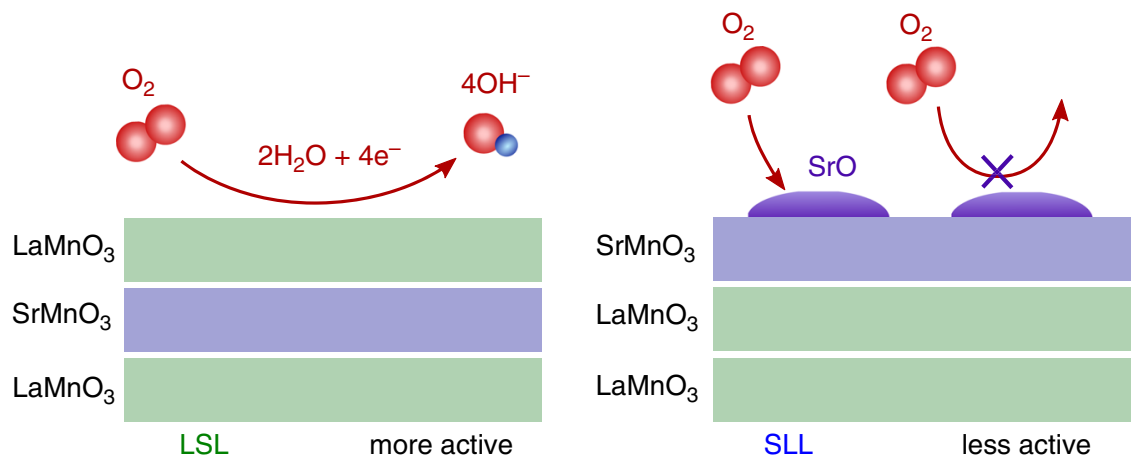

Fig. 4 Schematic of proposed mechanism of surface oxygen interaction. $\mathrm{LaMnO}_{3}-\mathrm{SrMnO}_{3}-\mathrm{LaMnO}_{3}(\mathrm{LSL})$ interacts with oxygen by adsorbing oxygen atoms without significantly changing surface structure. $\mathrm{SrMnO}_{3}-\mathrm{LaMnO}_{3}-\mathrm{LaMnO}$ (SLL) interaction with oxygen involves migration of the A-site $\mathrm{Sr}$ atoms to the surface to form Sr-O like bonds

placed in the sub-surface (LSL) compared with when Sr is placed on the surface (SLL). Stoerzinger et al. have shown that the charge transfer capability in $\mathrm{La}_{1-\mathrm{x}} \mathrm{Sr}_{\mathrm{x}} \mathrm{MnO}_{3}$ (measured using the [Fe $\left.(\mathrm{CN})_{6}\right]^{3-/ 4-}$ outer sphere redox reaction) depends on $\mathrm{x}$, the $\mathrm{Sr}$ content. Their results suggested that in addition to tuning the $e_{g}$ occupation, the $\mathrm{Sr}$ addition also benefits the ORR activity of $\mathrm{LaMnO}_{3}$ by providing carriers to increase conductivity ${ }^{30}$. Interestingly, we observe no difference in the charge transfer capabilities between LLS, LSL, and SLL, for the same $\left[\mathrm{Fe}(\mathrm{CN})_{6}\right]^{3-/ 4-}$ outer sphere redox reaction (Supplementary Figure 6). Therefore, the availability of transferrable electrons does not explain the ORR difference between SLL, LSL, and LLS (Fig. 2). The observation that $\left(\mathrm{LaMnO}_{3}\right)_{2} /\left(\mathrm{SrMnO}_{3}\right)$ is not limited by the availability of transferrable electrons is consistent with the transport measurements, which showed that $\left(\mathrm{LaMnO}_{3}\right)_{2} /$ $\left(\mathrm{SrMnO}_{3}\right)$ is metallic ${ }^{29,33,34}$.

APXPS analysis. With conductivity ruled out, we study the surface chemistry and electronic structure using APXPS. Supplementary Figure 7 shows the valence band spectra at lowenergy $(340 \mathrm{eV}$, inelastic mean free path, IMFP, $\sim 0.6 \mathrm{~nm})$ and high-energy (780 eV, IMFP, $\sim 1.3 \mathrm{~nm}$ ) excitations in both vacuum $\left(p \mathrm{O}_{2}<10^{-9}\right.$ Torr $)$ and near-ambient pressure $\left(p \mathrm{O}_{2} \sim 10^{-1}\right.$ Torr $)$. The valence spectra of $\mathrm{La}_{1-\mathrm{x}} \mathrm{Sr}_{\mathrm{x}} \mathrm{MnO}_{3}$ is principally assigned to a combination of the $\mathrm{O} 2 p(6 \mathrm{eV})$ and $\mathrm{Mn} 3 d$ states $(2 \mathrm{eV})^{35-38}$. As we move from deeper probing depth $(\sim 1.3 \mathrm{~nm})$ to shallower probing depth $(\sim 0.6 \mathrm{~nm})$, the valence spectra undergo a spectral weight transfer from the high-energy edge to about $2 \mathrm{eV}$ from the Fermi level. This change is attributed to the surface localization, which reduces the bandwidth and produces a localized, narrow Mn $3 d$ state at the surface. These experiments have two implications. First, the use of a molecular orbital model is a reasonable approximation for the oxide surfaces given the localized nature of the Mn $d$-state. Second, even with synchrotron APXPS, the difference in the valence band spectra between the superlattices are not resolvable. We have therefore focused our analysis on the core spectra, which have superior signal-to-noise ratios.

The core spectra provide insight into the surface chemistry difference between LSL and SLL. Background-subtracted spectra show that $\mathrm{La} 4 d$ peaks are insensitive of the $\mathrm{SrMnO}_{3}$ placement and the $\mathrm{pO}_{2}$ level (Supplementary Figure 8). This observation indicates that $\mathrm{La}$ is mostly a spectator and does not interact with atmospheric oxygen. We similarly observe that Sr $3 d$ peaks in LSL and LLS do not change with $p \mathrm{O}_{2}$, which is consistent with the idea that the sub-surface and sub-sub-surface Sr cannot "see" atmospheric oxygen (Supplementary Figure 9). However, for SLL, APXPS reveals that $\mathrm{Sr}$ can react with oxygen to form Sr-oxide-like species (Fig. 4, Supplementary Figure 9). This surface $\mathrm{Sr}$ component $(\sim 135 \mathrm{eV})$ grows systematically with $\mathrm{pO}_{2}$ (Fig. 5, see Supplementary Figure 9 and Supplementary Table 1 for spectra analysis). Surface segregation of Sr-oxide like species have been observed for $\mathrm{La}_{1-\mathrm{x}} \mathrm{Sr}_{\mathrm{x}} \mathrm{MnO}_{3}$ powders, pellets, and films ${ }^{39-41}$. We similarly believe that the top $\mathrm{SrO}$ layer within the superlattice could segregate past the top $\mathrm{MnO}_{2}$ layer to form Sr-oxide species on the surface of SLL. These observations suggest that the formation of the surface Sr-oxide could block the oxygen access to the surface $\mathrm{Mn}$ once the catalyst is exposed to atmospheric oxygen during the ORR. In this picture, LSL is more active than SLL because $\mathrm{SrMnO}_{3}$ resides in the sub-surface layer. Even though their surfaces nominally share the same local electronic structure, LSL surface is more accessible due to the absence of blocking Sr oxides.

Surface site availability for oxygen adsorption plays a critical role in ORR on Pt. Stamenkovic et al. have suggested that the surface site availability enhances the ORR activity on $\mathrm{Pt}_{3} \mathrm{Ni}(111)$ 10. We believe that a similar phenomenon occurs for the LSL heterostructure. The presence of $\mathrm{Sr}$ in $\mathrm{LaMnO}_{3}$ (i.e., $\mathrm{La}_{2 / 3}$ $\mathrm{Sr}_{1 / 3} \mathrm{MnO}_{3}$ ), although beneficial by modifying the $e_{\mathrm{g}}$ occupation of the Mn to increase the ORR activity, can simultaneously reduce the surface site availability by forming a surface Sr-oxide group that restricts the oxygen access to the active Mn sites. Strmcnik et al. have previously suggested that the surface oxide species could reduce the surface site availability by interacting with the ions in the electrochemical double layer ${ }^{42}$. This scenario is also possible in our superlattices.

Our result shows the double-edged effect of $\mathrm{Sr}$ in $\mathrm{La}_{2 / 3} \mathrm{Sr}_{1 / 3} \mathrm{MnO}_{3}$, where $\mathrm{Sr}$ can promote ORR by modifying the Mn electronic structure, but suppress the surface site availability when placed too close to the surface. The double-edged effect may explain why increasing the $\mathrm{Mn}$ valence state by oxygen overstoichiometry (e.g., $\mathrm{LaMnO}_{3+\delta}$ ) results in a more active catalyst than by aliovalent substitutions (e.g., $\mathrm{La}_{1-\mathrm{x}} \mathrm{Ca}_{\mathrm{x}} \mathrm{MnO}_{3}$ ), despite both leading to $\mathrm{Mn}$ catalysts with nearly the same $\mathrm{Mn}$ oxidation state and crystal structure ${ }^{20}$. We emphasize, however, that this is unlikely the only effect. As recently highlighted, Mn perovskite oxides are more active when synthesized using a coprecipitation method $20,43,44$. Defects and surface segregation likely play a critical role also. Finally, we note that we were unable to resolve major differences between the superlattices in either the valence band spectra, O $1 s$ (K-edge), or Mn $2 p$ (L-edge) $\mathrm{X}$-ray absorption (Supplementary Figures 10,11), which likely points to the need for higher resolution, higher efficiency X-ray techniques, especially new detector technologies that can resolve local electronic structure in the near-surface layer. Previous X-ray studies on $\mathrm{La}_{1-x} \mathrm{Sr}_{x} \mathrm{MnO}_{3}$ have shown only subtle changes in valence band for $0.3 \leq x \leq 0.6$ despite observable differences in 

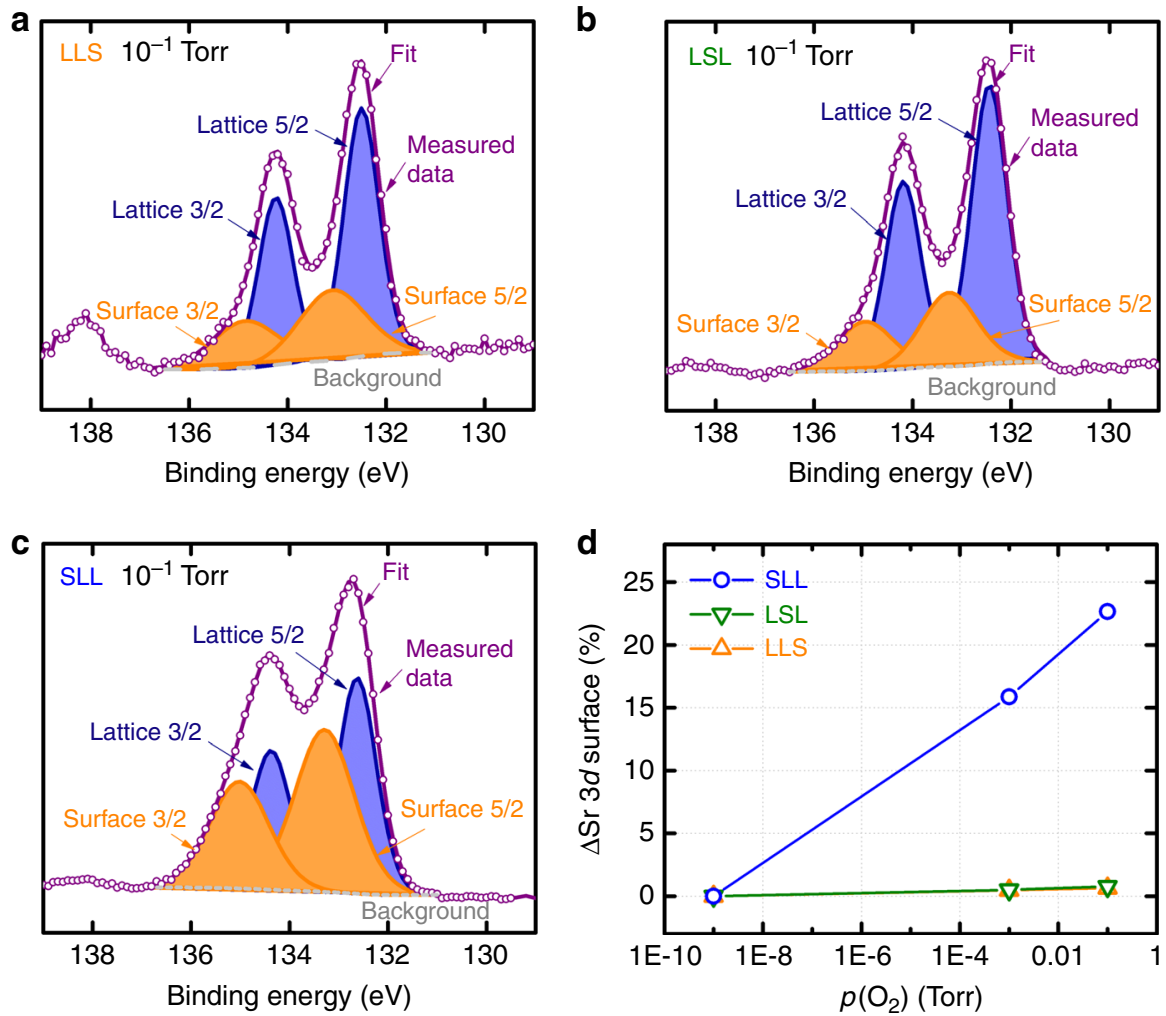

Fig. 5 Sr 3d ambient pressure X-ray photoelectron spectra and compositional fits. Sr 3d ambient pressure x-ray photoelectron spectroscopy (APXPS) background-subtracted spectra and compositional fits for a LLS, b LSL, and $\mathbf{c} S L L$ at $p\left(\mathrm{O}_{2}\right)$ at $10^{-1}$ Torr. The orange doublet corresponds to the surface $\mathrm{Sr}$ component, which we attribute to the Sr-oxide, whereas the blue doublet corresponds to the lattice Sr component within the bulk film. $\mathbf{d}$ The change in surface $\mathrm{Sr}$ component concentration $\left(p\left(\mathrm{O}_{2}\right)-10^{-9}\right.$ Torr) grows systematically with $p\left(\mathrm{O}_{2}\right)$ for SLL, whereas remain constant for LSL and LLS, suggesting the reaction of the surface $\mathrm{Sr}$ species in $\mathrm{SLL}$ upon contacting atmospheric oxygen. $\mathrm{LaMnO}_{3}-\mathrm{LaMnO}_{3}-\mathrm{LaMnO}_{3}$ is referred to as " $\mathrm{LLL}^{\prime}$, $\mathrm{LaMnO}_{3}$ - $\mathrm{LaMnO}{ }_{3}$ $\mathrm{SrMnO}_{3}$ as "LLS", $\mathrm{LaMnO}_{3}-\mathrm{SrMnO}_{3}-\mathrm{LaMnO}_{3}$ as "LSL", and $\mathrm{SrMnO}_{3}-\mathrm{LaMnO}_{3}-\mathrm{LaMnO}_{3}$ as "SLL"

electronic properties ${ }^{35,45}$. Developing finer resolution spectroscopy methods is an essential future step to reveal the nature of surface bonding in transition-metal oxides designed with customized surface and sub-surface.

\section{Discussion}

Our investigation of the surface and sub-surface customizations of $\mathrm{La}_{2 / 3} \mathrm{Sr}_{1 / 3} \mathrm{MnO}_{3}$ reveals that ORR is most active when $\mathrm{SrMnO}_{3}$ resides in the sub-surface vs. when it resides in the surface, the sub-sub-surface, or randomly distributed. APXPS reveals that this observation stems from the double-edged effect of Sr, which benefits ORR by modifying the electronic structure of the Mn sites, but can also form non-catalytic $\mathrm{Sr}$ oxides that reduce the surface site availability. Analysis of the valence band and elemental peak spectra in the presence of oxygen reveals that $\mathrm{Sr}$ modifies the electronic structure but also reacts to form a detrimental oxide species when present in the surface layer. Our finding demonstrates the Goldilocks effect, where moving the $\mathrm{Sr}$ closer to the surface allows the surface $\mathrm{Mn}$ atoms to more efficiently catalyze ORR, but $\mathrm{Sr}$ at the surface poisons the catalyst and reduces functionality. As a result, the catalyst is most active when the surface and sub-surface layer are $\mathrm{LaMnO}_{3}$ and $\mathrm{SrMnO}_{3}$, respectively. Most importantly, our work points to an opportunity to design an ORR catalyst by customizing surface and subsurface stoichiometry, orientation, and structure. The ability to control both parameters independently and rationally with atomic-layer precision can lead to the discovery of new catalysts with functionalities superior to those accessed through traditional bulk-composition control.

\section{Methods}

MBE. Epitaxial $\left(\mathrm{LaMnO}_{3}\right)_{2 n} /\left(\mathrm{SrMnO}_{3}\right)_{n}(n=1,2)$ superlattices were deposited on (001) $-\mathrm{SrTiO}_{3}$ single crystals using $\mathrm{MBE}$. $\mathrm{LaMnO}_{3}(\mathrm{LLL}), \mathrm{La}_{2 / 3} \mathrm{Sr}_{1 / 3} \mathrm{MnO}_{3}$ and five superlattices (LLS, LSL, SLL, LLSSLL, and SSLLLL) were prepared using a layer-bylayer deposition. In all synthesized heterostructures, the first letter represents the surface layer. All films were terminated with the Mn layer and XRD confirms the pseudo-cubic perovskite structure.

APXPS. To establish the connection between the surface and sub-surface customizations and electronic structure, we use APXPS ${ }^{46}$ to monitor the surface chemistry of the heterostructures. APXPS measurements were collected at Beamline 9.3.2 at the Advanced Light Source (ALS) of the Lawrence Berkeley National Laboratory (LBNL). After loading, all samples were heated to and held at $200^{\circ} \mathrm{C}$ at $1 \mathrm{mTorr} p \mathrm{O}_{2}$ for $30 \mathrm{~min}$ to remove surface adsorbed organics, which were monitored via the C1s spectra. We tune to the excitation energy such that the kinetic energy of the escaping electron is uniformly $250 \mathrm{eV}$ for all elemental peaks. This kinetic energy corresponds to a probing depth of around one functional unit $(\sim 1.1$ $\mathrm{nm}$ ). The following elemental peaks were measured (at the specified excitation energies): $\mathrm{O} 1 s(780 \mathrm{eV}), \mathrm{Sr} 3 d(385 \mathrm{eV})$, and $\mathrm{La} 4 d(340 \mathrm{eV})$. During the core peak measurements, we simultaneously collect the valence spectra. La $4 d$ XPS peaks were calibrated to the leading edge of the $\mathrm{La} 4 d$ peaks to be at $100 \mathrm{eV}$ as La peaks are qualitatively unaffected by changes in $p \mathrm{O}_{2}$ (Fig. S1). Other peaks were calibrated with respect to the La $4 d$ peaks of the same sample and pressure by aligning the $\mathrm{O}$ $2 p$ edge of the valence band spectra $(7-8 \mathrm{eV})$ to the calibrated valence band spectra of the La $4 d$ peaks with an error of $\pm 0.1 \mathrm{eV}$. We use a Shirley background correction. We also collected Mn L-edge and O K-edge X-ray absorption spectra in a partial-electron-yield mode in the same conditions as APXPS. All spectra were normalized to the background absorption below and above the absorption edge. 
Electrochemical characterizations. Electrochemical measurements were conducted in a standard three-electrode cell (Pine) using a potentiostat (Bio-Logic). The reference electrode was a $\mathrm{Ag} / \mathrm{AgCl}$ redox couple in a saturated $\mathrm{KCl}$ solution, calibrated to the $\mathrm{H}_{2} / \mathrm{H}^{+}$redox. The ohmic resistance correction used the highfrequency real-axis intercept of the impedance measurement. Front contacts were made using gallium-indium eutectic (Sigma-Aldrich, 99.99\%) and silver paint (Ted Pella, Leitsilber 200) for electrical contact. The contact and the sides and back of the substrate were all covered with epoxy (Omegabond 101) to ensure electrochemical insulation of these regions ${ }^{30}$. All samples were front contacted to avoid passing the current through the film-substrate interface. The ORR measurements were conducted in $\mathrm{O}_{2}$-saturated (Airgas, ultrahigh-grade purity) $0.1 \mathrm{M} \mathrm{KOH}$, prepared from Milli-Q water $(18.2 \Omega \cdot \mathrm{cm}$, Millipore) with KOH pellets (SigmaAldrich, $99.99 \%$ ) at a rate of $10 \mathrm{mV} / \mathrm{s}$. The ORR curves were obtained by subtracting the ORR current measured in $\mathrm{O}_{2}$ vs. background current measured in $\mathrm{Ar}$ (Airgas, ultrahigh-grade purity) in $0.1 \mathrm{M} \mathrm{KOH}$. The ORR activity is from the third cycle, where the subsequent scan did not vary by $>5 \%$ of the previous scan. The error bars represent the standard deviations for at least three independent measurements. The $\left[\mathrm{Fe}(\mathrm{CN})_{6}\right]^{3-/ 4-}$ measurements were conducted in Ar-saturated 0.1 $\mathrm{M} \mathrm{KOH}$ solution with $5 \mathrm{mM} \mathrm{K}_{4} \mathrm{Fe}(\mathrm{CN})_{6} \cdot 3 \mathrm{H}_{2} \mathrm{O}$ (Alfa Aesar, 98.5-102.0\%) and $\mathrm{K}_{3} \mathrm{Fe}$ $(\mathrm{CN})_{6}$ (Alfa Aesar, $99 \%$ ) at a rate of $10 \mathrm{mV} / \mathrm{s}$.

\section{Data availability}

The data that support the findings of this study are available from the authors on reasonable request.

Received: 16 March 2018 Accepted: 7 September 2018

Published online: 02 October 2018

\section{References}

1. Stamenkovic, V. R., Strmcnik, D., Lopes, P. P. \& Markovic, N. M. Energy and fuels from electrochemical interfaces. Nat. Mater. 16, 57-69 (2017).

2. Bruce, P. G., Freunberger, Sa, Hardwick, L. J. \& Tarascon, J.-M. Li-O2 and Li-S batteries with high energy storage. Nat. Mater. 11, 19-29 (2011).

3. She, Z. W. et al. Combining theory and experiment in electrocatalysis: insights into materials design. Science 355, eaad4998 (2017).

4. Shao, Y. et al. Making Li-air batteries rechargeable: material challenges. Adv. Funct. Mater. 23, 987-1004 (2013).

5. Stamenkovic, V. R., Mun, B. S., Mayrhofer, K. J. J., Ross, P. N. \& Markovic, N. M. Effect of surface composition on electronic structure, stability, and electrocatalytic properties of Pt-transition metal alloys: Pt-skin versus Ptskeleton surfaces. J. Am. Chem. Soc. 128, 8813-8819 (2006).

6. Wu, G. et al. Carbon nanocomposite catalysts for oxygen reduction and evolution reactions: from nitrogen doping to transition-metal addition. Nano Energy 29, 83-110 (2016).

7. Hong, W. T., Risch, M., Stoerzinger, K. A. \& Grimaud, A. Toward the rational design of non-precious transition metal oxides for oxygen electrocatalysis. Energy Environ. Sci. 8, 1404-1427 (2015).

8. Voiry, D. et al. Enhanced catalytic activity in strained chemically exfoliated WS2 nanosheets for hydrogen evolution. Nat. Mater. 12, 850-855 (2013).

9. Wang, J. X. et al. Oxygen reduction on well-defined core-shell nanocatalysts: particle size, facet, and Pt shell thickness effects. J. Am. Chem. Soc. 131, 17298-17302 (2009).

10. Stamenkovic, V. R. et al. Improved oxygen reduction activity on $\mathrm{Pt}_{3} \mathrm{Ni}(111)$ via increased surface site availability. Science 315, 493-497 (2007).

11. Greeley, J. et al. Alloys of platinum and early transition metals as oxygen reduction electrocatalysts. Nat. Chem 1, 552-556 (2009).

12. MenningC. A.. \& ChenJ. G.. Thermodynamics and kinetics of oxygen-induced segregation of $3 \mathrm{~d}$ metals in Pt-3d-Pt (111) and Pt-3d-Pt (100) bimetallic structures. J. Chem. Phys. 128, 164703 (2008).

13. Zhang, J. et al. Mixed-metal Pt monolayer electrocatalysts for enhanced oxygen reduction kinetics. J. Am. Chem. Soc. 127, 12480-12481 (2005).

14. Tymoczko, J. et al. Oxygen reduction at a Cu-Modi fied Pt (111) model electrocatalyst in contact with nafion polymer. ACS Catal. 4, 3772-3778 (2014).

15. Bandarenka, A. S. et al. Design of an active site towards optimal electrocatalysis: overlayers, surface alloys and near-surface alloys of $\mathrm{Cu} / \mathrm{Pt}$ (111). Angew. Chem. - Int. Ed. 51, 11845-11848 (2012).

16. Suntivich, J. et al. Surface composition tuning of Au-Pt bimetallic nanoparticles for enhanced carbon monoxide and methanol electro-oxidation. J. Am. Chem. Soc. 135, 7985-7991 (2013).

17. Sasaki, K. et al. Recent advances in platinum monolayer electrocatalysts for oxygen reduction reaction: scale-up synthesis, structure and activity of $\mathrm{Pt}$ shells on Pd cores. Electrochim. Acta 55, 2645-2652 (2010).
18. Cui, C., Gan, L., Heggen, M., Rudi, S. \& Strasser, P. Compositional segregation in shaped Pt alloy nanoparticles and their structural behaviour during electrocatalysis. Nat. Mater. 12, 765-771 (2013).

19. Stephens, I. E. L. et al. Tuning the activity of $\mathrm{Pt}(111)$ for oxygen electroreduction by subsurface alloying. J. Am. Chem. Soc. 133, 5485-5491 (2011).

20. Suntivich, J. et al. Design principles for oxygen-reduction activity on perovskite oxide catalysts for fuel cells and metal-air batteries. Nat. Chem. 3, 546-550 (2011)

21. Yuasa, M., Tachibana, N. \& Shimanoe, K. Oxygen reduction activity of carbon-supported $\mathrm{La}_{1-} \mathrm{Ca}_{\mathrm{x}} \mathrm{Mn}_{1-} \mathrm{F}_{\mathrm{y}} \mathrm{O}_{3}$ nanoparticles. Chem. Mater. 25, 3072-3079 (2013)

22. Gorlin, Y. \& Jaramillo, T. F. A bifunctional nonprecious metal catalyst for oxygen reduction and water oxidation. J. Am. Chem. Soc. 132, 13612-13614 (2010).

23. Osgood, H., Devaguptapu, S. V., Xu, H., Cho, J. \& Wu, G. Transition metal ( $\mathrm{Fe}, \mathrm{Co}, \mathrm{Ni}$, and $\mathrm{Mn}$ ) oxides for oxygen reduction and evolution bifunctional catalysts in alkaline media. Nano Today 11, 601-625 (2016).

24. Tulloch, J. \& Donne, S. W. Activity of perovskite $\mathrm{La}_{1-\mathrm{x}} \mathrm{Sr}_{\mathrm{x}} \mathrm{MnO}_{3}$ catalysts towards oxygen reduction in alkaline electrolytes. J. Power Sources 188, 359-366 (2009).

25. Cao, R., Lee, J. S., Liu, M. \& Cho, J. Recent progress in non-precious catalysts for metal-air batteries. Adv. Energy Mater. 2, 816-829 (2012).

26. Lima, F. H. B., Calegaro, M. L. \& Ticianelli, E. A. Investigations of the catalytic properties of manganese oxides for the oxygen reduction reaction in alkaline media. J. Electroanal. Chem. 590, 152-160 (2006).

27. Koster, G., Kropman, B. L., Rijnders, G. J. H. M., Blank, D. H. A. \& Rogalla, H. Quasi-ideal strontium titanate crystal surfaces through formation of strontium hydroxide. Appl. Phys. Lett. 73, 2920-2922 (1998).

28. Monkman, E. J. et al. Quantum many-body interactions in digital oxide superlattices. Nat. Mater. 11, 855-859 (2012).

29. Adamo, C. et al. Electrical and magnetic properties of $\left(\mathrm{SrMnO}_{3}\right)_{\mathrm{r}}\left(\mathrm{LaMnO}_{3}\right)$ 2n superlattices. Appl. Phys. Lett. 92, 112508 (2008).

30. Stoerzinger, K. A. et al. Highly active epitaxial $\mathrm{La}_{(1-\mathrm{x})} \mathrm{Sr}_{\mathrm{x}} \mathrm{MnO}_{3}$ surfaces for the oxygen reduction reaction: role of charge transfer. J. Phys. Chem. Lett. 6 $1435-1440$ (2015).

31. Dong, S. et al. Magnetism, conductivity, and orbital order in $\left(\mathrm{LaMnO}_{3}\right)_{2 \mathrm{n}} /$ $\left(\mathrm{SrMnO}_{3}\right)_{\mathrm{n}}$ superlattices. Phys. Rev. B - Condens. Matter Mater. Phys. 78, 1-4 (2008).

32. Nanda, B. R. K. \& Satpathy, S. Electronic and magnetic structure of the $\left(\mathrm{LaMnO}_{3}\right)_{2 \mathrm{n}} /\left(\mathrm{SrMnO}_{3}\right)_{\mathrm{n}}$ superlattices. Phys. Rev. B. 79, 054428 (2009).

33. Koida, T. et al. Effect of A-site cation ordering on the magnetoelectric properties in $\left[\left(\mathrm{LaMnO}_{3}\right)_{\mathrm{m}} /\left(\mathrm{SrMnO}_{3}\right)_{\mathrm{m}}\right]_{\mathrm{n}}$ artificial superlattices. Phys. Rev. B. 66, 1-6 (2002).

34. Bhattacharya, A. et al. Metal-insulator transition and its relation to magnetic structure in $\left(\mathrm{LaMnO}_{3}\right)_{2 \mathrm{n}} /\left(\mathrm{SrMnO}_{3}\right)_{\mathrm{n}}$ Superlattices. Phys. Rev. Lett. 100, 257203 (2008)

35. Saitoh, T. et al. Electronic structure of $\mathrm{La}_{1-\mathrm{x}} \mathrm{Sr}_{\mathrm{x}} \mathrm{MnO}_{3}$ studied by photoemission and x-ray-absorption spectroscopy. Phys. Rev. B. 51, 13942-13951 (1995)

36. Sarma, D. D. et al. Temperature-dependent photoemission spectral weight in $\mathrm{La}_{0.6} \mathrm{Sr}_{0.4} \mathrm{MnO}_{3}$. Phys. Rev. B 53, 6873-6876 (1996).

37. Chikamatsu, A. et al. Band structure and Fermi surface of $\mathrm{La}_{0.6} \mathrm{Sr}_{0.4} \mathrm{MnO}_{3}$ thin films studied by in situ angle-resolved photoemission spectroscopy. Phys. Rev. B 73, 195105 (2006).

38. Chainani, A, Mathew, M. \& Sarma, D. D. Electron spectroscopic investigation of the semiconductor-metal transition in La1-xSrxMnO3. Phys. Rev. B 47, 15397-15403 (1993).

39. Decorse, P., Caboche, G. \& Dufour, L. A comparative study of the surface and bulk properties of lanthanum-strontium-manganese oxides $\mathrm{La1}$-xSrxMnO3 $\pm \delta$ as a function of Sr-content, oxygen potential and temperature. Solid State Ionics 117, 161-169 (1999).

40. Monsen, A. F. et al. Surface stoichiometry of $\mathrm{La}_{0.7} \mathrm{Sr}_{0.3} \mathrm{MnO}_{3}$ during in vacuo preparation; a synchrotron photoemission study. Surf. Sci. 606, 1360-1366 (2012).

41. Fister, T. T. et al. In situ characterization of strontium surface segregation in epitaxial $\mathrm{La}_{0.7} \mathrm{Sr}_{0.3} \mathrm{MnO}_{3}$ thin films as a function of oxygen partial pressure. Appl. Phys. Lett. 93, 1-4 (2008).

42. Strmcnik, D. et al. The role of non-covalent interactions in electrocatalytic fuel-cell reactions on platinum. Nat. Chem. 1, 466-472 (2009).

43. Stoerzinger, K. A., Risch, M., Han, B. \& Shao-Horn, Y. Recent insights into manganese oxides in catalyzing oxygen reduction kinetics. ACS Catal. 5, 6021-6031 (2015).

44. Symianakis, E. et al. Electrochemical characterization and quantified surface termination obtained by low energy ion scattering and $\mathrm{X}$-ray photoelectron spectroscopy of orthorhombic and rhombohedral LaMnO3 powders. J. Phys. Chem. C. 119, 12209-12217 (2015). 
45. Horiba, K. et al. In vacuo photoemission study of atomically controlled $\mathrm{La}_{1-\mathrm{x}} \mathrm{Sr}_{\mathrm{x}} \mathrm{MnO}_{3}$ thin films: composition dependence of the electronic structure. Phys. Rev. B. 71, 155420 (2005).

46. Whaley, J. A. et al. Note: fixture for characterizing electrochemical devices inoperando in traditional vacuum systems. Rev. Sci. Instrum. 81, 086104 (2010).

\section{Acknowledgements}

We acknowledge Amit Bhatia, Runbang Tang, Chengyu Liu, and Kevin E. Fritz for their help with the X-ray measurements, and Elizabeth A. Nowadnick ('Beth'), Jason K. Kawasaki, and Julia A. Mundy for insightful discussions. This material is based upon work supported by the U.S. Department of Energy, Office of Science, Office of Basic Energy Sciences under Award Number DE-SC-0018029. This work made use of the Cornell Center for Materials Research (CCMR) Shared Facilities, which are supported through the NSF MRSEC program (DMR-1120296). The Advanced Light Source is supported by the Director, Office of Basic Energy Sciences, of the U.S. Department of Energy under contract no. DE-AC02-05CH11231. C.A. and D.G.S. acknowledge funding from the National Science Foundation under grant no. DMR-1610781. E.J.M. and S.J.M. acknowledge funding from the Army Research Office (W911NF-15-1-0133). Substrate preparation was performed in part at the Cornell NanoScale Facility, a member of the National Nanotechnology Coordinated Infrastructure (NNCI), which is supported by the National Science Foundation (grant ECCS-15420819).

\section{Author contributions}

C.J.E. and J.S. conceived of the idea for the experiment. C.A. and E.J.M. synthesized the heterostructures under the supervision of D.G.S. and S.J.M. C.J.E. and D.-Y.K. designed and carried out the electrochemical and X-ray experiments. All authors contributed to the analysis and to the writing of the manuscript.

\section{Additional information}

Supplementary Information accompanies this paper at https://doi.org/10.1038/s41467018-06503-8.

Competing interests: The authors declare no competing interests.

Reprints and permission information is available online at http://npg.nature.com/ reprintsandpermissions/

Publisher's note: Springer Nature remains neutral with regard to jurisdictional claims in published maps and institutional affiliations.

(c) Open Access This article is licensed under a Creative Commons Attribution 4.0 International License, which permits use, sharing, adaptation, distribution and reproduction in any medium or format, as long as you give appropriate credit to the original author(s) and the source, provide a link to the Creative Commons license, and indicate if changes were made. The images or other third party material in this article are included in the article's Creative Commons license, unless indicated otherwise in a credit line to the material. If material is not included in the article's Creative Commons license and your intended use is not permitted by statutory regulation or exceeds the permitted use, you will need to obtain permission directly from the copyright holder. To view a copy of this license, visit http://creativecommons.org/ licenses/by/4.0/.

(C) The Author(s) 2018 